\title{
LA DIVERSIDAD DE CAPACIDADES PARA APRENDER, UN VALOR A SER RECONOCIDO EN LAS AULAS
}

\section{THE DIVERSITY OF LEARNING SKILLS, A SITUATION TO BE RECOGNIZED IN THE CLASSROOM}

\author{
Paula Andrea Restrepo García \\ Universidad de Manizales \\ prestrepo@umanizales.edu.co \\ Marly Liliana Erazo Bermúdez \\ Institución Educativa El Boquerón, \\ marlybre@hotmail.com \\ Franqui Idelva Ramírez Plaza \\ Institución Educativa Agrícola Luis \\ Nelson Cuellar \\ idelvara1@hotmail.com
}

\author{
Ruth Mary Vélez Correa \\ Institución Educativa Técnico Tunía \\ rumvelez@gmail.com \\ Andrés Alexander Yanza \\ Institución Educativa Mojarras \\ andreyanzabravo@gmail.com
}

\begin{abstract}
Resumen: Este artículo es resultado de la investigación denominada Significados y sentidos de la diversidad de capacidades de aprendizaje que han configurado en su contexto educativo los niños, las niñas y jóvenes del grado sexto de la Institución Educativa (IE) El Boquerón, en el municipio de Timbío del departamento del Cauca (Colombia) ${ }^{1}$. Metodológicamente esta investigación es cualitativa de corte etnográfico, en la cual se usaron diversas técnicas como: la entrevista no estructurada, los talleres grupales y las cartas asociativas.Los resultados indican que si bien los estudiantes reconocen la diversidad de capacidades que los caracteriza como una cualidad que los hace únicos, también señalan que las prácticas educativas, altamente competitivas y selectivas, no les permiten explorar al máximo su potencialidad, frustrando en muchos casos las posibilidades de éxito escolar.
\end{abstract}

Palabras clave: Diversidad, capacidades, aprendizajes, significados, sentidos, diferencias escolares, etnografía.

\begin{abstract}
This article is the result of the investigation named "Senses and meanings for the diversity" made with a mixed group of boys and girls of 6th grade of the I.E. El boqueron, located in the municipality of Timbio, Department of Cauca (Colombia). Methodologically, this investigation is qualitative type with ethnographic approach using various techniques such as; associative letters, unstructured interview and group workshops. The results indicate that although the students recognize the diversity of skills like a quality that makes them unique, they also note that highly competitive and selective educational practices do not allow them to explore their full potential, frustrating in many cases the chances of school success.
\end{abstract}

Key words: Diversity, capabilities, learning, meanings, senses, school differences, ethnography.

1 El estudio hace parte de un proyecto de investigación nacional denominado "Sentidos y significados de la diversidad: perspectivas para una educación incluyente en la región andina, amazónica y pacífica de Colombia desde las voces de los niños, niñas y jóvenes” vinculado al Grupo de Investigación Educación y Pedagogía: Saberes, Imaginarios e Intersubjetividades, en la línea de... 


\section{Introducción.}

El siguiente artículo condensa los planteamientos y hallazgos producto de la investigación denominada Significados y sentidos de la diversidad de capacidades de aprendizaje que han configurado en su contexto educativo los niños, las niñas y los jóvenes del grado sexto de la Institución Educativa (IE) El Boquerón, en el municipio de Timbío del departamento del Cauca (Colombia), cuyo propósito central se orienta a reconocer sus voces y posturas en el proceso de movilización hacia prácticas inclusivas.

El estudio parte de la idea de la diversidad asociada a las diferencias, que como características naturales configuran a los seres humanos; por ello es importante abordarla desde los espacios escolares con el fin de movilizar prácticas que garanticen procesos inclusivos. Por este motivo surgió la necesidad de investigar respecto a los significados y sentidos que construyen los estudiantes de la IE EI Boquerón (TimbíoCauca, Colombia), respecto a la diversidad de capacidades.

Nos enfocamos en la diversidad de capacidades al reconocer la importancia que tiene identificarla en los educandos, pues cada uno de ellos posee diferentes ritmos y estilos de aprendizaje, que exigen movilizar prácticas incluyentes como oportunidad de construir conocimiento e interactuar con los otros en medio de las diferencias. Para analizar estos aspectos, se tuvieron en cuenta planteamientos como los de Ferreiro (2001: 3), cuando afirma que:

"Desde su misma fundación, la escuela pública de las nuevas naciones, como institución, tuvo dificultades para trabajar con la diversidad. A pesar de los esfuerzos de la institución escolar, las diferencias subsistian. Las primeras diferencias que fueron claramente reconocidas fueron las diferencias individuales en el rendimiento escolar".

Aquellas diferencias individuales exigen atender la diversidad de capacidades de los estudiantes, para desarrollar los procesos de aprendizaje comprendiendo que cada sujeto tiene un estilo particular de aprender, el cual se debe identificar y potenciar en los escenarios escolares. De este modo, cobra trascendencia indagar por los sentidos y los significados construidos por los educandos respecto a la diversidad de capacidades, con el fin de movilizar prácticas educativas sustentadas en el reconocimiento de las diferencias.

Dichos sentidos y significados son abordados como construcciones sociales que los sujetos configuran de acuerdo a las características sociales, familiares y culturales que los conforman. Analizar dichas significaciones contribuye a asumir la diversidad como "una cualidad propia de la vida: no existe cosa más natural que la diversidad. La diferencia es lo normal" (López, 2004: 50). Por ello, la escuela como establecimiento social ha de esforzarse por cumplir con formar para la vida y dar ejemplo en el reconocimiento de la diversidad como valor.

Finalmente, cabe decir que este estudio tiene relevancia puesto que contribuye a indagar cómo significan los educandos la diversidad de capacidades y a partir de ello, generar praxis educativas que posicionen a los estudiantes como agentes de

... investigación en Desarrollo Humano. Investigadoras principales: Paula Andrea Restrepo García, Claudia Esperanza Cardona López y Claudia Cárdenas Zuluaga, Docentes-Investigadoras de la Facultad de Ciencias Sociales y Humanas de la Universidad de Manizales. El proyecto fue desarrollado como requisito de grado de los estudiantes de la Maestría en Educación desde la Diversidad de la Universidad de Manizales (Colombia). 
cambio que asumen la diversidad como una oportunidad de encontrarse con el otro y reconocerse en medio de las diferencias y de la pluralidad de capacidades que cada sujeto posee.

Además, a partir de las construcciones que hacen los niños, niñas y jóvenes respecto a la diversidad, es posible configurar nuevas comprensiones acerca de la realidad de su entorno, del reconocimiento y aceptación del otro con sus diferencias, sus dificultades y sus capacidades, reduciendo la tendencia de la escuela a considerar a los estudiantes por sus capacidades de aprendizaje como "buenos y malos", para hacer de la institución un lugar de encuentro de las diferencias donde todos y cada uno viva y conviva con la diversidad, haciendo de ella una realidad tan natural como la vida.

\section{Objetivo}

Comprender los significados y sentidos construidos por algunos estudiantes de la Institución Educativa El Boquerón (Cauca, Colombia), respecto a la diversidad capacidades para aprender, con el fin de movilizar prácticas que los posicionen como sujetos que asumen la diversidad como un valor agregado y una oportunidad de reconocerse con los otros en medio de las diferencias.

\section{Antecedentes}

Las investigaciones realizadas en la última década sobre diversidad de capacidades apuntan hacia algunos ejes centrales: estilos y ritmos de aprendizaje y reconocimiento de la diversidad de los educandos, con el fin de encaminarse hacia prácticas pedagógicas incluyentes que garanticen la permanencia y el bienestar de los educandos en los escenarios escolares.

Estudios como los de Jiménez, Álvarez, Gil, Murga y Téllez (2006) y Simón (2011) establecen que, para propiciar el respeto por la diversidad de capacidades que caracteriza a los educandos, es necesario que los currículos escolares estén en constante evaluación para que sean flexibles y se ajusten a las necesidades de cada uno de los estudiantes; también resaltan la importancia de conocer las competencias previas de los educandos y, en particular, el estilo de aprendizaje predominante en cada uno de ellos, para posibilitar procesos de enseñanza y aprendizaje pertinentes y eficaces en función de sus capacidades.

El estudio de Simón (2011), plantea que las estrategias que el estudiante pone en juego para aprender son indicadores de sus preferencias, lo cual corrobora que dos personas en las mismas circunstancias responden de modo diferente permitiendo la identificación de estilos de aprendizaje dominantes.

El análisis realizado por Jiménez et al. (2006), se acerca al conocimiento de las percepciones, actitudes y valores de los alumnos "con más capacidades". Esta línea de investigación permitió identificar que en la escuela, quienes se muestran con "más capacidades", permanecen en el aula, mientras aquellos identificados "con menos capacidades" por lo general desertan o permanecen en lugares segregados, sin motivación alguna para mantenerse en el sistema. También se plantea que suelen persistir estereotipos de género, en especial contra las mujeres con capacidades sobresalientes. 
Otras investigaciones, como las realizadas por Mora y Herrera (2003) y Blanco (2008), analizan el interés por los significados que otorgan los profesores a la diversidad, tras el análisis de la cotidianidad que se vive en el aula de clase. Son estudios de tipo cualitativo, que intentan conocer mediante observaciones sistemáticas el grupo de estudiantes, pero especialmente al docente en el quehacer de su profesión, y su perspectiva como actor que reconstruye el sistema de representaciones sociales en sus prácticas individuales.

En el mismo sentido, estudios realizados por Rosano (2008), Velázquez (2010) y Gómez (2012), exponen la importancia de promover una educación inclusiva que oriente sus procesos de enseñanza y aprendizaje desde la diversidad del estudiantado; así por ejemplo, manifiestan que es necesario generar una cultura escolar incluyente, lo cual exige cambios desde los equipos directivos, para proponer estrategias que conlleven a promover el reconocimiento por la diversidad y que responda a las diferencias de los estudiantes sin ningún tipo de segregación.

Los estudios señalados tienen como propósito indagar cuáles son las condiciones que deben existir en las escuelas para propiciar una educación inclusiva, debido a que muchos niños, niñas y jóvenes no tienen posibilidad de participar de la escuela, debido a que habitan en condiciones geográficas que limitan su acceso o porque viven en situaciones de pobreza que les impide participar de los procesos escolares. Por ello, las investigaciones refieren la importancia de iniciar proyectos educativos basados en la equidad y dispuestos a evitar la exclusión para garantizar la democracia y la permanencia de los estudiantes en el aula de clase.

Estudios similares reiteran la importancia de atender la diversidad en los espacios escolares, tal como lo afirman Blanco (2008) y Almeida, Coral y Ruíz (2014), al señalar que para lograr que los estudiantes piensen de manera crítica, es necesario que se respeten sus diferencias y se parta de las potencialidades que cada uno de ellos posee, para identificar las estrategias pedagógicas más convenientes en cada uno de los casos que requieran los educandos.

Por lo tanto, las investigaciones sugieren que las instituciones educativas, no pueden centrar su atención únicamente en el éxito académico del estudiantado, sino que deben prestar mayor interés por los aspectos humanos que valoren la diversidad que caracteriza a cada uno de ellos.

Lo mencionado requiere un cambio profundo en las escuelas para que todos los miembros que la conforman estén encaminados hacia prácticas incluyentes, flexibles ante la diversidad y que garanticen la participación de todos los educandos en las prácticas pedagógicas, para asegurar calidad educativa y equidad social. Los estudios analizados también reiteran la importancia que tiene el hecho de que los docentes asignen valor a la diversidad, para que posibiliten dentro del aula de clase el reconocimiento de las diferencias como aspectos positivos que enriquecen las relaciones entre los sujetos.

Finalmente, otros estudios consultados tuvieron por objetivo analizar la actitud de los docentes en formación y de los estudiantes universitarios respecto a la diversidad de los sujetos; análisis como los de Alpuín, González y Pérez (2005), Alonso, Navarro y Vicente (2008) y Solórzano (2013), permiten identificar que muchos docentes son sensibles a la diversidad pero sienten como un desafío permanente el hecho de atender una población estudiantil tan heterogénea. Las investigaciones señalan que es fundamental que desde las universidades se oriente a los futuros docentes y a los educandos respecto a la importancia de reconocer la diversidad de los su- 
jetos para garantizar praxis educativas incluyentes que no admitan ningún tipo de discriminación.

Los antecedentes consultados nos permiten de alguna forma comprender los siguientes aspectos: en primer lugar, es indispensable que las competencias previas de los estudiantes sean conocidas por sus docentes así como sus estilos de aprendizaje, teniendo en cuenta que estos se constituyen en insumos indispensables para promover procesos de enseñanza y aprendizaje significativos. En segundo lugar, se evidencian en la cotidianidad escolar prácticas explícitas de exclusión de los estudiantes connotados como "con menos capacidades" en el aula, a través de la invisibilización y la falta de acompañamiento adecuado para promover su motivación y garantizar el éxito escolar. Finalmente, permiten reflexionar respecto a la importancia de que los maestros, desde sus formaciones universitarias asuman la diversidad como un aspecto natural y favorable para enriquecer las interacciones entre los sujetos.

\section{Abordaje Teórico}

\subsection{Construcción de sentidos y los significados: expresados a través del lenguaje.}

Los sentidos y los significados se pueden abordar desde múltiples perspectivas; en este caso, partiremos desde una óptica constructivista, la cual los asume como construcciones mentales que los seres humanos configuran respecto a una palabra, una situación o un fenómeno. Al respecto, algunos autores como Vigotsky (1995), afirman que los seres humanos siempre construyen significados y sentidos de acuerdo a su nivel de pensamiento, interpretación, descubrimiento, en relación con sus saberes previos y con la cultura que los rodea. Por ello es evidente que los educandos construyen significados, de acuerdo al entorno en el que habitan y a los saberes familiares y sociales con los cuales se hayan configurado.

Para Vigotsky (1995), los sentidos se construyen de acuerdo a las experiencias, a la cultura y a las formas sociales; mientras que el significado tiende a ser mucho más estable y se asocia a un término más literal. Se deduce de este concepto vigotskiano, que las expresiones orales de los educandos, mediante las cuales dan cuenta de los significados y sentidos que han construido acerca de la diversidad de capacidades de aprendizaje, responden al proceso mismo de formación de su lenguaje interiorizado.

Desde esta perspectiva, los sentidos construidos por los educandos respecto a la diversidad de capacidades, guardan relación con la formación que ellos han adquirido desde sus hogares y que se ha complementado en los espacios sociales y escolares. Esta construcción de sentidos y significados se materializa con el lenguaje, puesto que a través de éste se expresan las ideas, los pensamientos y la cultura.

Los sentidos y los significados son coherentes con las condiciones contextuales de los educandos y se expresan a través del lenguaje que "se considera como la codificación de un "potencial de conducta" en un "potencial de significado", es decir, como un medio de expresar lo que el organismo humano "puede hacer", en interacción con otros organismos humanos, transformándolo en lo que "puede significar" (Halliday, 1994: 33). Por lo tanto, los significados que los educandos construyan respecto a la diversidad de capacidades se manifestarán en las expresiones que ellos empleen al referirse al asunto, puesto que el significado es "la generalización de la palabra en la relación con los otros" (Ballesteros, 2005: 232). 
Para Ballesteros (2005), los significados no se construyen de manera individual sino que están subordinados a la interacción con los otros. Así mismo, para Lederach (citado por Ballesteros, 2005: 234):

"La construcción de significado tiene que ver con el proceso de dar sentido a algo y se logra al relacionar ese algo con otras cosas ya conocidas, además el cambio de significado requiere una función de re-encuadre o re-enmarque definido como un proceso mediante el cual algo se reubica y se relaciona con cosas diferentes".

De tal manera que las construcciones de sentidos y significados pueden cambiar de acuerdo a las construcciones sociales; por ello cobra valor el hecho de que en la escuela se movilicen prácticas incluyentes pues estas permitirán que las significaciones que tienen los miembros de las instituciones educativas respecto a la diversidad, en este caso particular, la diversidad de capacidades, se asocien a posturas que reconozcan la diversidad como un valor agregado en los sujetos.

\subsection{La diversidad: como derecho, valor y característica genuina de los seres humanos.}

Antes de referirse a la diversidad de capacidades, es necesario, en primera medida, abordar el término diversidad de manera general. Muchos autores lo asocian con la capacidad que poseen los seres humanos de ser distintos; otros lo abordan como las características de las personas de ser diferentes; y otros lo relacionan con condiciones naturales de los sujetos. Todas estas percepciones son válidas, dado que la diversidad es un asunto tan natural como la vida y por ello debe asumirse de la misma manera, con espontaneidad, sin ningún tipo de prejuicio o limitante.

Para Gimeno (1995), la diversidad así comprendida, debe ser asumida desde la escuela puesto que es una forma de fomentar una cultura de ética, equidad y respeto por las diferencias; el autor plantea que si bien es cierto que las escuela en muchas ocasiones no puede atender la desigualdad escolar, tampoco es posible que la estimule; para ello sugiere cambios en la estructura del sistema educativo con diseños de currículos flexibles y democráticos que correspondan a la pluralidad del alumnado.

Del mismo modo, López (2001), expone argumentos que respaldan la postura de asumir la diversidad como un aspecto genuino en los individuos; el autor afirma que en una sociedad en la cual se vulneran con frecuencia los derechos humanos, es apremiante la participación de la escuela como organismo que contribuya a evitar el rechazo o la segregación de los sujetos. La escuela entonces debe contribuir a evitar los procesos de enseñanza homogéneos para crear una cultura de la diversidad.

Desde esta mirada, la diversidad se aborda de manera significativa y no como un asunto de moda, lo cual exige cambiar los pensamientos y las prácticas pedagógicas homogenizantes por actitudes que reconozcan las diferencias como valor. Esto implica docentes reflexivos, dispuestos a orientar los aprendizajes partiendo de las particularidades de cada educando y sobre todo, listos a cambiar de paradigmas educativos que les impidan considerar la diversidad como un aspecto a valorar. De tal manera, que según Santos (2005), no es posible la educación sino se permite a los educandos desarrollarse de acuerdo a sus posibilidades, y si no se desarrollan procesos educativos que promuevan la sensibilidad hacia las diferencia humanas.

Es tarea de la escuela generar una cultura de la diversidad la cual permita que existan entre los docentes y los educandos prácticas relacionales dialógicas que faciliten la interacción, el aprendizaje y el reconocimiento de las diferencias. Promover dicha cultura exige currículos flexibles, evaluaciones de acuerdo al ritmo de los estudiantes 
y la participación de todos los miembros de la comunidad educativa en la lucha por el respeto por las diferencias.

\subsection{La diversidad de capacidades en el contexto escolar}

En la actualidad la educación inclusiva ha tomado mucha importancia en el contexto educativo y se sugieren diversas estrategias que pueden usar los docentes para hacer de la inclusión una práctica real; dentro de ellas es preciso señalar el reconocimiento de las diferentes capacidades que poseen los educandos. De acuerdo con Rodríguez, (2012), al hablar de diversidad en los contextos escolares, es necesario insistir en la necesidad del reconocimiento por parte de los docentes de la diversidad de capacidades, su comprensión en profundidad y su aplicación en el quehacer pedagógico. Es preciso reconocer que el concepto es complejo y pocos docentes saben sus alcances y cómo aplicarlo, por lo tanto, las instituciones educativas deben aceptar el reto de su divulgación y enseñanza.

Rodríguez (2012: 4), también señala que la diversidad, entendida como "una característica de la conducta y de la condición humana", puede ser asumida como una oportunidad para reconocer al otro y valorar las distintas capacidades que posea. Por ello es conveniente que desde los escenarios escolares se entienda que los educandos son distintos respecto a su manera de pensar, aprender, actuar y realizar determinadas actividades. De igual manera, dichas capacidades pueden ser modificadas de acuerdo a su motivación intrínseca o extrínseca; son dinámicas dadas las cualidades psíquicas complejas del cerebro.

Dichos aspectos han de ser atendidos en las prácticas pedagógicas para evitar exigir a todos los educandos que aprendan de manera homogénea, puesto que no existen dos sujetos que piensen o actúen de igual forma frente a una misma situación y las oportunidades que se le puedan ofrecer en la vida son recibidas por cada quien, en función de cómo ha construido su propia individualidad.

Desde una perspectiva similar, Arnaiz (2010) también reitera la importancia de reconocer las diferencias individuales que deben ser tenidas en cuenta a la hora de construir conocimiento en la escuela. Ella considera importante educar "desde la igualdad, en el respeto del otro, del diferente, del que procede de otra cultura, hable otra lengua, practique religión o no, padezca una discapacidad física o psíquica y sufra el rechazo por vivir en situaciones de pobreza y marginación social" (Arnaiz, 2010: 3).

Así mismo, de Devalle y Vega (2006: 7) consideran que el respeto y la atención a la diversidad se encuentran entre los pilares de la educación, para promover una nueva sociedad inclinada hacia el pluralismo democrático; por lo cual afirman que "negar la diferencia es no reconocer la diversidad con la pretensión de imponer la uniformidad" (Devalle y Vega, 2006: 7); al imponerse lo homogéneo, se suprime la autonomía y prevalece la intolerancia, lo cual resta dinámica al deseo del descubrimiento del otro y de sus diferencias.

Por lo tanto, es menester que la educación se oriente desde lo heterogéneo, tal como lo recomienda Levine (2002), al mencionar la necesidad de generar acercamientos con los niños, las niñas y los jóvenes para observar cuáles son sus potencialidades, sus actuaciones y sus preferencias, sin sentir la mirada juzgadora del adulto. Esta aproximación también permite identificar cuáles son las verdaderas necesidades educativas y encaminarlas hacia el fortalecimiento de las cualidades y el aminoramiento de los defectos para evitar el fracaso escolar. 


\subsection{Inteligencias múltiples y aprendizaje diverso.}

Operar en un contexto tan complejo como el educativo, demanda aceptar que la inteligencia se manifiesta de múltiples formas que deben ser reconocidas en los espacios escolares. Respecto a ello, Gardner (1983: 111), cuestionó "la validez de determinar la inteligencia de un individuo por medio de la práctica de sacar a una persona de su ambiente de aprendizaje natural y pedirle ciertas tareas aisladas que nunca había hecho antes y probablemente, nunca elegiría volver a hacer". Por ello Gardner, se percató de la necesidad de crear una nueva teoría a la que llamó de las inteligencias múltiples, mediante la cual se propuso ampliar los alcances del potencial humano, al asociar la inteligencia con "la resolución de problemas y la creación de un ambiente que represente un contexto rico y de actividad natural". (Gardner, 1983: 112).

En cuanto al aprendizaje diverso, hay que recordar que las formas de enseñanza y aprendizaje de los sujetos están configuradas por las condiciones biológicas, sociales, económicas, étnicas, políticas y culturales en las que está inmerso el alumno; estas aspectos no pueden ser impedimento para que el alumno aprenda; tal como lo señala Baquero (2006), todo sujeto tiene formas naturales y capacidades para aprender. No se puede afirmar que alguna persona no pueda hacerlo, todos tienen posibilidades; y el hecho de que los alumnos no puedan lograr un aprendizaje satisfactorio en las áreas específicas, en los tiempos y en los modos que el docente requiere, no significa que no haya logrado un aprendizaje básico, pues así como en algunas actividades tiene dificultades y le resulta complejo, en otras puede mostrar grandes capacidades que ejecuta con facilidad.

En este sentido, "los estilos de aprendizaje son los rasgos cognitivos, afectivos y fisiológicos, que sirven como indicadores relativamente estables, de cómo los discentes (el que aprende; el estudiante) perciben, interaccionan y responden a sus ambientes de aprendizaje" (Alonso y Gallego, 1994, citados por Simón, 2011: 181). Cada persona puede manifestar diversas formas de aprender; por ello es vital que los docentes determinen los estilos de aprendizaje de sus estudiantes, con el fin de propiciar ambientes apropiados a la condición particular de cada uno de ellos.

Según Woolfolk (2006), al hablar de "estilo de aprendizaje", se está haciendo referencia a que cada individuo para aprender, recurre a un método o conjunto de estrategias con los cuales se le facilita hacerlo. También, de acuerdo con lo que se quiere aprender, la persona busca las estrategias más adecuadas, mostrando ciertas tendencias que permiten identificar un estilo de aprendizaje predominante, revelando sus preferencias hacia determinados tipos de medios de representación, que pueden ser: visuales, auditivos, kinestésicos o combinaciones de ellos. En este sentido, Gimeno (1995), también advierte que la escuela debe luchar para romper con la uniformidad y reconocer los distintos ritmos de aprendizaje y las necesidades de los alumnos.

Así, se reitera el valor de una práctica pedagógica orientada hacia el reconocimiento de los distintos estilos de aprendizaje de los estudiantes y el diseño de currículos flexibles que garanticen procesos de aprendizaje incluyentes y sobre todo, con oportunidades de permanencia en la escuela y respeto por los derechos de todos los educandos. 


\section{Metodología}

El presente es un estudio de tipo cualitativo con enfoque etnográfico realizado con la participación de 34 estudiantes con edades que oscilan entre los 11 y 16 años, del grado sexto de la Institución Educativa El Boquerón en el municipio de Timbío (departamento del Cauca, Colombia).

Los estudios etnográficos según Goetz y LeCompte (1998: 28), permiten emplear datos fenomenológicos y analizar una situación específica, en este caso representada por las significaciones construidas por los educandos de la I.E. El Boquerón, en el municipio de Timbío (Cauca, Colombia), respecto a la diversidad de capacidades.

El trabajo etnográfico, desde la perspectiva de Guber (2004: 36), debe generar un retrato "vivido de los más variados aspectos de una cultura: economía, organización social y política, sistema religioso y de creencias, formas de socialización de los jóvenes, tratamiento de los ancianos, vínculos con la naturaleza, relaciones con otros grupos culturales, arte, tecnología, entre otros". Esto posibilita la cercanía de los investigadores al contexto escolar pues permite frecuentes encuentros con los participantes, con el fin de cumplir con el objetivo de una investigación, que en este caso, es vislumbrar cómo los educandos están semantizando la diversidad de capacidades.

Para obtener información utilizamos técnicas etnográficas como la entrevista no estructurada y la observación participante. También se realizaron cartas asociativas que permitieran identificar las significaciones que los niños, niñas y jóvenes otorgaban a la diversidad.

Se consideró oportuno este tipo de entrevistas porque surgen de manera espontánea, rompiendo un poco el formalismo con los participantes, para facilitar la formulación de las preguntas y la familiaridad con el investigador. También se empleó la observación participante que posibilita sistematizar los discursos y las acciones de las personas e "interactuar con los sujetos observados" (Hernández, Fernández y Baptista, 2003: 428).

Para complementar la recolección de información se realizaron las cartas asociativas (Abric, 1994), que son una técnicas que permite, a partir de un término inductor, asociar nuevas palabras de manera libre, donde se pueden identificar lazos significativos con el término seleccionado, lo que implica la definición de conceptos y categorías inducidas, de modo que, al interactuar con el grupo específico de estudiantes, las representaciones emerjan y se hagan visibles.

Algunos estudiantes realizaron adicionalmente un registro fotográfico por las instalaciones de la institución para identificar lo que para ellos representaba la diversidad de capacidades. También se realizaron talleres grupales en los cuales los educandos pudieron elaborar carteleras exponiendo los conceptos que tenían respecto a la diversidad, para socializarlo con los demás compañeros.

\section{Hallazgos.}

\subsection{La diversidad como variedad propia de la naturaleza.}

Dado que el objetivo de este estudio era indagar por las semánticas construidas por los educandos respecto a la diversidad de capacidades, se examinó en primera medida lo que ellos identificaban, opinaban o creían respecto a la diversidad en general. 
En primer lugar, muchos estudiantes asociaron el término diversidad a distintos aspectos que los rodean. Así por ejemplo, de acuerdo a las cartas asociativas realizadas por los estudiantes de grado sexto, se encontró que algunos de ellos asocian el término diversidad, a palabras como animales, refiriéndose tanto a los de su entorno como a los que han observado en libros, revistas o la televisión y que de alguna forma les ha llamado la atención. También lo asociaron a términos como diversión, variedad, naturaleza, plantas, bosques, libertad y personas. Ninguno de ellos relacionó el vocablo diversidad con diferencias o distintas capacidades de los sujetos.

En las cartas asociativas también los estudiantes destacaron que la diversidad es una posibilidad de disfrutar de todo lo que está disponible en su entorno, lo contrario conduce a "amargarse la vida". Asumen que todos los elementos de la naturaleza son diferentes y también la manera en la cual se relacionan, por ejemplo, plantas y animales con personas y los animales entre sí.

\subsection{La diversidad como diferencia sexual: de la primera de las diferencias.}

En las entrevistas realizadas con los educandos, se identificaron diversas posturas de algunos de ellos respecto a la diversidad, en relación a las diferencias sexuales. De esta forma, al preguntarles acerca de las maneras de relacionarse con personas de otro sexo, los estudiantes expresan que les gusta interactuar con sus compañeras, pero dejan entrever ciertos estereotipos de género asociados por ejemplo a los tópicos de conversación como se afirma a continuación:

"Prof.: ¿tú piensas que son diferentes los niños de las niñas? Pues hay unos que sí; porque es que...este... los niños pues hablan de otras cosas y las niñas solo es así hablar como de novios así. Prof.: ¿Y cuando forman grupos con ellas, digamos las integran o cada cual por su lado? OR: Así pues cada uno se integra...las niñas se hacen es ellas mismas, hacen grupo es entre ellas para hablar de cosas que a nosotros no nos gustan, son temas de niñas un poquito ehhh... como aburridores" (Oscar Ruiz, 12 años).

Así, se evidencia de alguna manera una visión de las mujeres como colectivo o grupo homogéneo, "idénticas entre sí" y, en este caso, opuestas a los hombres. Las diferencias sexuales también fueron evidentes cuando se aborda el tema de los éxitos académicos; en este sentido, los estudiantes indicaban que muchas veces les molestaba que las niñas obtuvieran buenas notas, como lo expresa uno de ellos:

"BR: Ah ya, algunas veces, pero no todos los del salón somos así, o sea pues, los niños sobre todo siempre critican mucho cuando la mujer va a hablar, por lo menos a Paola y Alexa las critican mucho porque son las que siempre cumplen, sacan siempre cincos y hasta izan bandera, entonces a uno como que no le gusta eso, porque siempre las niñas, entonces no más eso, de lo contrario las niñas a los hombres no". (Brayan Ramírez, 12 años).

En la expresión anterior, se destaca el hecho de relacionar el cumplimiento, la responsabilidad y la participación con cualidades que permiten garantizar el éxito académico y que además se asocia de forma directa con características propias de la mujer. Aún así, al parecer el desempeño destacado de una mujer genera cierta incomodidad entre los compañeros hombres quienes a través de críticas las ponen en cuestión En este sentido, se percibieron tensiones entre los significados y sentidos construidos respecto a la diversidad como diferencia sexual, puesto que los estudiantes en algunos casos se referían a ellas de manera favorable pero también hacían explícita la idea de que reconocen las diferencias porque "toca", como si fuera una obligación y no un asunto natural. 
Igualmente se indagó por aspectos relacionados con la orientación sexual ante lo cual se evidenciaron algunas respuestas como la siguiente:

"Prof:: ¿Que piensas tú de las relaciones entre hombre y hombre, o mujer y mujer? LC: Umm, uyy no, (se ríe y se tapa la cara), noo, ahí si mal, creo que mal. Prof.: Mal por qué.: LC: Pues ellos tienen sus gustos, allá ellos si les gusta los hombres o las mujeres, pero no me parece" (Leidy Cifuentes, 13 años).

Lo mencionado evidencia de alguna forma falta de aceptación y reconocimiento de los educandos por la diversidad sexual, pues se observa cierta postura moralista y de sanción ante la situación. Otros estudiantes destacan en este caso el respeto y la aceptación como una obligación ante las diferentes orientaciones sexuales, identificándose que especialmente las niñas se muestran respetuosas por dicha condición y tratan de manera cordial a sus compañeros a pesar de las diferencias:

"YM: Creo que toca que aceptarlo, porque todos somos diferentes y piensan diferente. Por ejemplo, Francisco, se llamaba el que decían que era gay, él era muy bueno en lo que hacía y sacaba muy buenas notas. Él ocupaba siempre el primer puesto" (Yerson Muñoz, 14 años).

\subsection{Diversidad de capacidades: entre el reconocimiento y la indiferencia}

Al interrogar a los estudiantes sobre las capacidades de aprendizaje, algunos afirmaron que aunque son consientes de que tienen muchas capacidades para aprender, a veces se sienten con limitaciones para entender ciertos temas, lo cual les genera incomodidad respecto al resto del grupo, tal como lo afirma uno de los entrevistados:
“Prof: ¿tu crees que todos los niños y las niñas tienen diferentes capacida- des para aprender? JR: Yo creo que sí profe, todos podemos, aunque bueno a veces uno no es que se las sepa todas, a mi a veces me va bien en matemáticas digámoslo así, pero a veces con la profe de español...mmm no tanto, aunque uno pues se supone que tiene capacidades a veces le queda durito y los demás del grupo como que se le burlan, yo no sé..." (Juan Camilo Rodríguez, 13 años).

En este relato se puede identificar como los estudiantes reconocen no tener las mismas habilidades e intereses para todas las áreas, poniendo en evidencia una idea de desarrollo de cierta forma disincrónico. Igualmente se resalta como las dificultades experimentadas por algunos estudiantes en muchas ocasiones se convierten en motivo de burla entre los pares ante lo cual se denota estructuras mucho más individualistas que colaborativas.

Los estudiantes también manifestaron que sienten que a medida que avanzan en sus estudios se enfrentan a temas más complejos que les exigen mayores esfuerzos; por ejemplo, hay algunos que se sienten incómodos estudiando matemáticas mientras otros consideran que la forma en la cual el profesor explica les resulta difícil de entender y que eso ha generado cierta apatía hacia el área:

\footnotetext{
“Prof: ¿por qué dices que se te dificultan las matemáticas? LS: Es que profe mire por ejemplo, el profe Manuel, el de matemáticas, el explica rapidito y dice que uno debe poner atención porque él no va a volver a llenar el tablero con ejercicios... y eso es lo que hace, llena ese tablero que mejor dicho, yo a veces no entiendo ni mu... por eso a mi las matemáticas si como que no me entran..." (Leidy Suarez, 12 años).
}

Los discursos de los educandos permiten vislumbrar que algunos métodos de enseñanza de los docentes son tradicionalistas y por tanto orientados a un grupo ideal- 
mente homogéneo, lo cual limita el reconocimiento por parte del profesor de los distintos ritmos, estilos y capacidades de los educandos. Los estudiantes también manifestaron muchos deseos por sobresalir en el colegio, pero sienten que sólo algunos estudiantes tienen éxito académico mientras que los otros simplemente cumplen los logros propuestos de manera básica:

“Prof: ¿crees que te va bien académicamente en tu colegio? YM: pues no sé, así que yo ocupe el primer puesto y esas cosas no profe. Es que mire le explico, por ejemplo en mi salón siempre izan la bandera Sebastián y Yesica, uno ya sabe, hasta los profesores ya saben, así que uno así se mate estudiando no va a lograr eso, uno pasa ahí raspadito" (Yeison Molina, 14 años).

Desde esta perspectiva, es evidente que en muchas circunstancias en las aulas de clase se generan divisiones entre los estudiantes que tienen éxito académico y los que se limitan a observar como los otros ocupan los primeros puestos; según las expresiones de los educandos, los docentes en ocasiones avalan y fomentan esas divisiones y siempre ofrecen el apoyo a los estudiantes que logran mejores notas, como lo expresa uno de los alumnos:

"Prof: ¿tú crees que los profesores valoran las capacidades de todos los niños? LM: pues cómo así profe, ah ya le entiendo ¿que si los profes nos ayudan así a todos? Yo creo que no, porque ellos son contentos con los amigos de uno así juiciosos, que hacen las tareas y así les va bien, pero conmigo por ejemplo, me dicen, ia ver Laura!, ¿usted cuando será que va a izar bandera? porque aquí ya uno sabe siempre quienes son los mejores, entonces profe uno así pues ya sabe que nunca se parará allá a que le pongan la bandera" (Laura Maca, 13 años).

De este modo los docentes asumen una postura favorable ante los educandos que evidencian mejor desempeño académico pero se limitan un poco con aquellos que no manifiestan los mejores resultados. Además, muchos profesores ignoran o no hacen uso de estrategias pedagógicas que les permitan identificar las capacidades que los alumnos poseen para encontrar formas apropiadas que faciliten el aprendizaje de todos los estudiantes desde las diferencias que los caracterizan.

Los estudiantes también expresaron que aunque hay asignaturas o temáticas que les resultan un poco complejas, ellos son consientes de que si realizan esfuerzos y estudian con responsabilidad pueden ser capaces de lograr lo que se propongan; aceptan que tienen capacidades distintas y que eso es un aspecto natural que se manifiesta en el aula de clase:

“Prof.: ¿Notas en tu salón que algún niño tiene más capacidades que otro?

VR: Si, pero igualmente todos tenemos la capacidad, pero unos las utilizamos más que otros, no pues es normal. (Valentina Ruiz, 12 años). "Prof.: ¿Todos tienen las mismas habilidades o tienen habilidades diferentes? PC: No, diferentes, hay unas habilidades muy diferentes, por ejemplo Santiago Dorado él es muy bueno dibujando y los demás casi no, hay otros que son buenísimos en otras cosas pero..." (Paula Chito, 16 años).

Los educandos son consientes de la diversidad de capacidades que los caracteriza y lo asumen de manera natural, reconociendo que cada uno tiene habilidades especiales que los hacen únicos y capaces de aprender cualquier temática. Por ello es fundamental analizar dicha postura, pues si los estudiantes asumen la diversidad de capacidades como un asunto positivo, los docentes deberían sumarse a dicha posición para abordar las prácticas pedagógicas desde la diversidad como un valor 
agregado que enriquece la construcción de conocimiento y beneficia la autoestima en los educandos.

\title{
6.4. Desempeño académico como factor de reconocimiento, posicionamiento y competencia en las aulas.
}

En cuanto al rendimiento académico relacionado con la diversidad de capacidades, los estudiantes mencionan que algunas falencias respecto a lo académico surgen porque las prácticas pedagógicas les resultan desagradables y sienten que deben competir con los demás compañeros para tener reconocimiento, no solo en la escuela sino en la familia, como se expone a continuación:

\begin{abstract}
"Prof: ¿tienes buen desempeño académico? FR: pues más o menos, no mucho... Prof: ¿y eso por qué? FR: porque a mi casi no me gusta el estudio, me parece como un poquito aburridor. Además el estudio a veces es difícil y en la casa a uno le dicen que le debe ir bien pero la competencia es dura (risas)..." (Fernando Rodríguez, 12 años).
\end{abstract}

Aparte de ello, los alumnos que tienen éxito académico reciben reconocimiento público y se posicionan de manera favorable ante la escuela y la familia, mientras los que no reciben dicho mérito solo intentan aprobar en cierta medida lo que les orientan. Esta situación evidencia que solo un grupo de alumnos tiene la posibilidad de ser reconocido, los demás aparentemente no tienen cualidades para merecerlo:

\begin{abstract}
"Prof: ¿sientes que eres reconocido en la escuela por algún aspecto académico? AB: Yo no, nunca he ocupado el primer puesto. Prof: ¿de qué forma reconocen el desempeño académico? AB: pues por ejemplo, cuando uno es buen estudiante en el patio hacen la izada de bandera y lo sacan delante de todos los alumnos. Prof: ¿y que debes hacer para merecer ese reconocimiento? AB: Nooo pues mucho, eso es imposible, hay que saber harto y ser el mejor en todo $y$ no..." (Ángela Bravo, 13 años).
\end{abstract}

Se puede apreciar como los estudiantes se convencen de que ellos no cumplen con los requisitos para ser valorados en la escuela, lo cual refleja la falta de reconocimiento que existe por la diversidad de capacidades, puesto que si se considerara al educando desde sus potencialidades particulares, todos merecerían ser reconocidos de acuerdo a las habilidades que manifiesten en determinada área del conocimiento.

Surge entonces la demanda de docentes que actúen como facilitadores de oportunidades, dispuestos a identificar las virtudes de los educandos, para viabilizar múltiples formas de acceder al conocimiento, de forma tal que la utilización de estrategias pedagógicas sea coherente con la diversidad del estudiantado para evitar una competencia perjudicial en las aulas de clase y transformarla por relaciones dialógicas de ayuda mutua.

La competencia evidente en las aulas genera exclusión, pues los estudiantes que no alcanzan el éxito académico no tienen la oportunidad de ser reconocidos o posicionados ante los otros como los mejores. Esto también genera que la experiencia escolar para los educandos esté acompañada de exigencias y frustraciones puesto que siempre deben poner a prueba sus capacidades al ritmo que la escuela ordene en comparación permanente con sus pares. Esto se evidencia en el relato de un estudiante:

"Prof: ¿crees que en el aula de clase hay que competir por ser el mejor? CP.: pues... yo creo que sí porque si no, uno no podría ocupar el primer puesto, por ejemplo yo que gano el segundo puesto, pues me toca duro, y eso que estudio 
bastante y no puedo alcanzar o pasar a Yesica que a veces ocupa el primero." (Camilo Pérez, 13 años).

Los docentes por su parte, centran gran parte de sus expectativas en los logros de sus estudiantes, y esto a veces conlleva a que ignoren otros aspectos de los alumnos como las capacidades que ellos puedan poseer para otras áreas. Por ejemplo, el docente de matemáticas ignora si el estudiante es muy capaz en escritura o en dibujo, tal como se expresa a continuación:

“Prof: ¿cómo te va en el área de matemáticas? AG: a mi muy mal (risas). Siempre saco mala nota, casi no me gusta esa materia. Profe: entonces, ¿cuál materia te gusta? AG: Me gusta bastante artística porque la profe es chévere y soy bueno para el dibujo, siempre que ella hace concursos los gano, hasta un día gané al nivel del pueblo. Profe: y por ejemplo, ¿tu profesor de matemáticas sabe que tú tienes ese talento? Noooo profe, el profe que va a saber no ve que en matemáticas no se dibuja..." (Andrés Guerrero, 12 años).

Lo mencionado evidencia que en ocasiones no se abordan las capacidades que tienen los estudiantes para determinada área, sino que se espera que aprendan todo lo que se les oriente, sin tener en cuenta que poseen múltiples capacidades y que en ocasiones tienen más talento para ciertas actividades que para otras. Esta situación conlleva a que los estudiantes se sientan inferiores ante los que aprenden de manera más rápida aquellas áreas más valoradas en el ámbito académico, como lo afirma uno de ellos:

\footnotetext{
"Prof.: ¿En qué has notado que alguno de tus compañeros o compañeras sean más inteligente que tú? PC: Ella es como más creativa, no sé ella, ella como que inventa las cosas más y yo, yo hago las cosas así por hacer, en cambio ella se ingenia unas cosas más que yo todavía, ella es muy ordenada en cambio yo soy así, más o menos, ni tan ordenada ni tan desordenada, eso." (Paula Chito, 16 años)
}

Son variadas las expresiones de los estudiantes cuando afirman que otros compañeros tienen más capacidades que ellos, lo cual les genera frustración al sentirse con menos cualidades para aprender y afecta su autoestima puesto que se limita el despliegue de la diversidad de capacidades de los educandos. En términos generales, se identificó que el buen desempeño académico de los estudiantes es proporcional al reconocimiento que ellos recibirán en la escuela, por lo cual se minimizan los méritos y el posicionamiento de los educandos como sujetos capaces y con oportunidades para demostrar las múltiples habilidades que poseen en la construcción del conocimiento.

\section{Discusión}

Si se entiende la diversidad como "una característica de la conducta y de la condición humana" (Rodríguez, 2012:4), sería natural que se reconociera en todos los aspectos de la vida, sobre todo en los escenarios escolares; no obstante, de acuerdo a los planteamientos de los educandos, la diversidad, especialmente la de capacidades, muchas veces no es identificada ni valorada en el aula de clase, lo cual atenta contra las diferencias naturales de los sujetos, teniendo en cuenta que:

"Un nuevo modelo educativo que ha de construirse sobre la base de la comprensión de que todas las personas que acuden a la escuela son competentes para aprender. Aceptar este principio es iniciar la construcción de un nuevo discurso 
educativo al considerar la diferencia en el ser humano como un valor y no como defecto y a partir de ahí renacerá una cultura escolar que al respetar las peculiaridades e idiosincrasia de cada niño evitará las desigualdades, una cultura de una escuela sin exclusiones." (López, 2005: 15).

En las expresiones de los estudiantes se percibe cómo la diversidad de capacidades de aprendizaje no es estimulada por los docentes, puesto que como se identificó en los resultados, existen divisiones entre los estudiantes con buenos resultados académicos y los que no cumplen con todos los requisitos exigidos en cada una de las áreas del conocimiento.

Lo anterior posibilita que los estudiantes que no obtienen buenas calificaciones se vean opacados por quienes sí lo logran, lo cual exige que en las instituciones educativas se generen espacios para potenciar las capacidades de cada individuo y aportar al reconocimiento de su diversidad, puesto que aunque los estudiantes la significaron como algo inherente a su entorno, también describieron situaciones en las cuales la descalificación y el rechazo hacen presencia en su ámbito escolar.

De este modo, se pone en evidencia la necesidad de praxis educativas incluyentes que asuman la diversidad como valor, para que contribuyan, a que aquellos significados que se construyen respecto a ella y que han sido aparentemente inamovibles, cambien y den paso a una sociedad inclusiva que asuma las diferencias como oportunidades de encuentro con los otros.

Igualmente se identifica la demanda de espacios escolares que se conviertan en lugares que potencien las distintas capacidades de los educandos y que cuenten con docentes dispuestos a identificar la diversidad de cada uno de ellos para facilitarles la construcción colaborativa de conocimientos.

Según Baquero (2006), existen formatos escolares muy rígidos y homogéneos que impiden el reconocimiento de las distintas capacidades de los educandos, por lo cual expone la importancia de generar espacios escolares más flexibles y coherentes con las necesidades y la diversidad de cada uno ellos. Como plantea Restrepo (2015: 101):

"En este proceso de transformación de la visión de la razón de ser de la escuela, seguimos permitiéndonos olvidarnos de los sujetos, de sus particularidades, de sus formas únicas de habitar y construir sus proyectos vitales, y tendemos a homogeneizar nuestra comprensión del ser estudiante en promedios que necesariamente excluyen, relegan y marginan. Esta tendencia nos remite a pensar mucho más en la igualdad, la homogeneidad, la generalidad, y nos hace olvidar la unicidad, la heterogeneidad, la singularidad propia de lo vivo y específicamente de lo humano".

En esta dirección, Nussbaum (2012: 55), señala que "las capacidades pertenecen, en primer y prioritario lugar a las personas individuales, y solo luego, en sentido derivado, a los colectivos", por lo tanto, es necesario atender las particularidades de cada sujeto, como agente aportante a un colectivo, como se da en la escuela.

De esta manera cada estudiante merece una atención específica que garantice el desarrollo de sus capacidades particulares para evitar que, cómo lo expresaron los estudiantes, sean solo algunos quienes reciben méritos por las cualidades académicas que manifiestan, mientras los otros no tienen posibilidad de demostrar o desarrollar sus talentos por causa de estrategias pedagógicas tradicionales y homogéneizantes. 
Al respecto, Levine (2003) afirma que tanto la familia como los profesores deben ser diligentes en la búsqueda de talentos que posea cada educando y en los apoyos que requieran para superar cualquier dificultad de aprendizaje: "Nunca es tarde para comprender una mente y fortalecerla" (Levine, 2003: 57). En síntesis, las estrategias pedagógicas deben sustentarse en la idea de que cada estudiante tiene distintas capacidades y necesidades educativas, y que no por ello, unos son mejores que otros, simplemente son diversos y eso los hace merecedores de atención y valoración de sus diferencias.

\section{Conclusiones y Recomendaciones}

Los educandos de la IE El Boquerón, del municipio de Timbío (Departamento del Cauca, Colombia), han construido significados respecto a la diversidad de capacidades de aprendizaje, que reflejan, por una parte, los desafíos a los que ellos se enfrentan para acceder al conocimiento y por otra parte, la preocupación y los sentimientos que expresan al notar que el éxito académico solo pertenece a algunos estudiantes.

En este contexto, aprender de manera rápida y con facilidad algunas áreas específicas, es visto por los estudiantes como un recurso para mostrar sus capacidades y recibir méritos académicos; no obstante muchos de ellos manifestaron que se les dificulta sobresalir académicamente, lo cual les genera frustración y les limita motivarse para superar los obstáculos relacionados con el aprendizaje.

Los significados reconocidos por los estudiantes con relación a la diversidad de capacidades, se asocian a la idea de que los profesores esperan que todos aprendan lo mismo, de la misma manera y al mismo ritmo, por lo cual los educandos se sienten incómodos frente a su propia posibilidad de superación porque saben de antemano que solo algunos corresponderán a las exigencias del educador. Esto se genera ante prácticas educativas homogéneas que desconocen la diversidad de los educandos, por lo cual es necesario contar con docentes dispuestos a motivar y potenciar las distintas capacidades que poseen los alumnos.

De acuerdo con lo anterior, es necesario replantear las prácticas educativas que se desarrollan en la IE EI Boquerón (Cauca, Colombia), teniendo en cuenta que los sentidos y los significados que los estudiantes construyen respecto a la diversidad de capacidades permite apreciar situaciones que no contribuyen a la eficiencia y calidad de los procesos de enseñanza y aprendizaje, sino que evidencian prácticas pedagógicas desarrolladas por algunos docentes que ignoran la diversidad de estilos de aprendizaje de los estudiantes, dando lugar a situaciones discriminatorias.

Por lo tanto, se recomienda a futuros investigadores, continuar analizando los significados que construyen no solo los educandos, sino todos los miembros de las comunidades educativas frente a la diversidad de capacidades, para movilizar acciones conjuntas que permitan el diseño de currículos que consideren las diferencias de los educandos como una oportunidad para interactuar con los otros y potenciar en cada sujeto las capacidades que posee.

\section{Bibliografía}

Abric, J. C. (1994). Prácticas sociales y representaciones. México: Ediciones Coyoacán.

Almeida, M; Coral, F. y Ruíz, S. (2014). Didáctica Problematizadora para la configuración del Pensamiento Crítico en el marco de la atención a la diversidad. (Tesis inédita de 
Maestría). Universidad de Manizales, Colombia. Recuperado de http://ridum.umanizales.edu.co:8080/jspui/bitstream/6789/1727/1/TESIS\%20DE\%20GRADO.pdf

Alonso, M. J.; Navarro, R. y Vicente, L. (2008). Actitudes Hacia la Diversidad en Estudiantes Universitarios. Ponencia presentada en las Jornades de Foment de la Investigación en la Universitat Jaume. Cataluña, España. Recuperado de http://www.uji.es/bin/ publ/edicions/jfi13/44.pdf

Alpuín, G; González, M. A. y Pérez, M. (2005). Creencias y Actitudes de los estudiantes del último Curso del Magisterio Hacia la Atención a la Diversidad. Ponencia presentada en las Jornades de Foment de la Investigación en la Universitat Jaume. Cataluña, España. Recuperado de http://www.uji.es/bin/publ/edicions/jfi11/10.pdf

Arnaiz Sánchez, P. (2010). Sobre la atención a la diversidad. Universidad de Murcia. Murcia, España. Prensa Universitaria.

Ballesteros, B. P. (2005). El concepto de significado desde el análisis del comportamiento $y$ otras perspectivas. Universitas Psychologica, $N^{\circ} 4$, Vol.2, pp. 231-244.

Baquero, R. (2006). Sujetos y aprendizaje. Argentina: Ministerio de Educación, Ciencia y Tecnología de la Nación.

Blanco, P. M. (2008). La Diversidad en el Aula: Construcción de Significados que otorgan los profesores de Educación Parvularia, Enseñanza Básica y de Enseñanza Media, al trabajo con la Diversidad en una escuela municipal de la comuna de la Región Metropolitana. (Tesis inédita de maestría). Universidad de Chile. Recuperado de www.tesis. uchile.cl/handle/2250/106653?show=full.

Devalle de Rendo, A. y Vega, V. (2006). Una escuela en y para la diversidad: El entramado de la diversidad. Buenos Aires: Aique Grupo Editores.

Ferreiro, E. (2001). Pasado y presente de los verbos leer y escribir. Fondo de Cultura Económica. España.

Gardner, H. (1983). Frames of mind. The theory of multiple intelligences. Basic Books. New York: Paidós.

Gimeno Sacristán, J. (1995) Diversos y también desiguales. ¿Qué hacer en educación? Kikirikí. Revista del Movimiento Cooperativo Escuela Popular, №38, pp.18-25.

Goetz, J. y LeCompte, M. (1998). Etnografía y diseño cualitativo en investigación educativa. España: Ediciones Morata.

Gómez, I. (2012). Dirección escolar y atención a la diversidad: rutas para el desarrollo de una escuela para todos. (Tesis Doctoral inédita) Universidad de Huelva, España. Recuperado de http://rabida.uhu.es/dspace/handle/10272/5435

Guber, R. (2004). El salvaje metropolitano. Reconstrucción del conocimiento social en el trabajo de campo. Buenos Aires: Paidós.

Halliday, M. (1994). El lenguaje como semiótica social. La interpretación social del lenguaje y del significado. México: Fondo de Cultura económica.

Hernández Sampieri, R; Fernández Collado, C. y Baptista Lucio, P. (2003). Metodología de la investigación. México: McGraw-Hill.

Jiménez, C; Álvarez, B; Gil, J; Murga, M. y Téllez, J. (2006). Educación, diversidad de los más capaces y estereotipos de género. RELIEVE, №2, Vol.2. Recuperado de www. uv.es/RELIEVE/v12n2/RELIEVEv12n2_5.htm 
Levine, M. (2003). Mentes Diferentes, Aprendizajes Diferentes. Un modelo educativo para desarrollar el potencial individual de cada niño. España: Paidos.

López Melero, M. (2001). Cortando las amarras de la escuela homogeneizante y segregadora. Revista de Educación Nº 3, pp. 15-53.

López Melero, M. (2004). Construyendo una escuela sin exclusiones. Una forma de trabajar con proyectos en el aula. Málaga: Aljibe Editores.

López Melero, M. (2005). La ética y la cultura de la diversidad en la escuela inclusiva. Revista Electrónica Sinéctica, $N^{\circ} 29$, pp. 4-18

Mora, B. y Herrera, E. (2003). La búsqueda de la cotidianidad en el aula: Una perspectiva etnográfica de un quinto año de una Institución privada de la Dirección Regional de Educación de San Carlos. (Tesis inédita de Maestría). Universidad Estatal a Distancia de Costa Rica. Recuperado de repositorio.uned.ac.cr/.../La\%20busqueda\%20de\%20 la\%20cotidianidad.

Nussbaum, M. (2012). Crear capacidades: propuesta para el desarrollo humano. Barcelona. Paidós.

Restrepo, P. A. (2015). Pensando pedagogías de las diferencias: una posibilidad de reconocer en la educación la esencia de lo humano. En Corporación Universitaria Lasallista (Ed.) Retos y realidades de la psicología educativa. Memorias del Congreso Nacional de Psicología Educativa (ASCOFAPSI) (pp.99-112). Caldas: Editorial Lasallista.

Rodríguez Rodríguez, J. A. (2012). La diversidad del alumnado: Por diferentes capacidades y ritmos de aprendizaje. Escuela de Magisterio de la Universidad Complutense de Madrid. Prensa universitaria.

Rosano Ochoa, S. (2008). El camino de la inclusión educativa en punta hacienda (comunidad campesina de la sierra andina ecuatoriana). (Tesis inédita de Maestría). Universidad Internacional de Andalucía, España. Recuperado de http://dspace.unia.es/ bitstream/handle/10334/34/0050_Rosano.pdf?sequence=1

Santos Guerra, M. A. (2005). El dromedario no es un camello defectuoso. Atención a la diversidad en las instituciones educativas. TABANQUE, N¹9, pp.203-228.

Simón Simón, V. (2011). Diversidad de estilos de aprendizaje en el aula de música de ESO. Ensayos. Revista de la Facultad de Educación de Albacete, N²6, pp.179-195.

Solórzano, M. J. (2013) Escala Actdiv para medir la actitud hacia la diversidad. Revista Electrónica Actualidades Investigativas en Educación. Revista Actualidades Investigativas en Educación, Vol.13, N¹, pp.1-26.

Velázquez Barragán, E. (2010). La importancia de la organización escolar para el desarrollo de escuelas inclusivas. (Tesis Doctoral inédita). Universidad de Salamanca, España. Recuperado de http://gredos.usal.es/jspui/bitstream/10366/76601/1/DDOMI_ Velazquez_Barragan_E_Laimportanciadlaorganizacionescolar.pdf

Vigotsky, L. S. (1995). Pensamiento y lenguaje. Buenos Aires, Argentina: La Pléyade.

Woolfolk, A. (2006). Psicología educativa. México: Prentice Hall. 